Ueslei Teodoro 1

Thaís Gomes Verzignassi Silveira 1

Demilson Rodrigues dos Santos 2

Elcio Silvestre dos Santos 2

Ademar Rodrigues dos Santos 2

Otílio de Oliveira 2

João B. Kühl 1

Dayane Alberton 2

\section{Influência da reorganização, da limpeza do peridomicílio e a da desinsetização de edificações na densidade populacional de flebotomíneos no Município de Doutor Camargo, Estado do Paraná, Brasil}

\author{
Influence of rearrangement and cleaning of the \\ peridomiciliary area and building disinsectization \\ on sandfly population density in the municipality \\ of Doutor Camargo, Paraná State, Brazil
}

1 Departamento de Análises Clínicas, Universidade Estadual de Maringá. Av. Colombo 5790 , Maringá, $P R$ 87020-900, Brasil. uteodoro@uem.br 2 Núcleo de Entomologia de Maringá, Secretaria de Saúde do Paraná. Rua Gastão Vidigal 55, Maringá, $P R$ 87040-050, Brasil.

\begin{abstract}
Phlebotomine sandfly captures were performed in the domicile, domestic animal shelters, and peridomicile before and after rearrangement, cleaning, and disinsectization. Phlebotomine sandflies were captured with Falcão traps from October 1998 to April 2000 in the Recanto Marista district, Doutor Camargo municipality, Paraná State. The results were compared with those from October 1996 to September 1997. In this survey, 122,616 phlebotomine sandflies were captured using 10 traps, as compared to 157,983 in the previous period, with 6 traps. Lutzomyia neivai predominated in both periods. During the former period an hourly average of 1,641.9 phlebotomine sandflies were captured as compared to 806.7 in the latter. The results with four traps installed in the same locations shows that the hourly average was 1,318.8 in the former period and 156.0 in the latter. The large reduction in the number of phlebotomine sandflies captured in the second period may be due to the impact of alterations in the environment and the disinsectization of the buildings after the first period.
\end{abstract}

Key words Psychodidae; Leishmaniasis; Vector Control

Resumo Comparam-se os resultados de coletas de flebotomíneos, feitas com armadilhas de Falcão, no domicílio e em abrigos de animais domésticos no peridomicílio, antes e após a reorganização, a limpeza e a desinsetização destes ambientes. As coletas foram realizadas de outubro de 1998 a abril de 2000, no Recanto Marista, Município de Doutor Camargo, Estado do Paraná. Os resultados foram comparados com outros obtidos no período de outubro de 1996 a setembro de 1997. Nesta investigação foram coletados 122.616 flebotomíneos, com dez armadilhas, contra 157.983 coletados anteriormente, com seis armadilhas. Lutzomyia neivai predominou nos dois períodos de coletas. No primeiro foram coletados em média por hora (MH) 1.641,9 flebotomíneos contra 806,7 no segundo. Os resultados das coletas com quatro armadilhas, instaladas nos mesmos ecótopos, mostram que a MH no primeiro período foi 1.318, 8 e no segundo 156,0. Os dados sugerem que a redução expressiva no número de flebotomíneos coletados foi devido ao impacto das alterações introduzidas no ambiente e pela desinsetização das edificações, realizadas após o primeiro período de coleta.

Palavras-chave Psychodidae; Leishmaniose; Controle de Vetores 


\section{Introdução}

A leishmaniose tegumentar (LT) é endêmica no Brasil tendo sido notificados 418.179 casos no período de 1980 a 1999 (Lima, 2000; MS, 1997, 2000). Neste período, na Região Sul do país ocorreram 6.277 casos, representando $2,0 \%$ dos casos do Brasil. Destes, 6.264 (99,8\%) foram notificados no Estado do Paraná (Lima, 2000). Nesse Estado, a LT é conhecida desde o início do século passado, com notificação de casos até a década de 50, voltando a ser notificada em 1980. A LT persiste de forma endêmica no Estado do Paraná, com casos em 276 municípios dos 399 existentes, especialmente nas regiões Norte e Oeste (Lima, 2000).

Os abrigos de animais domésticos construídos muito próximos das habitações humanas, a ausência de boas condições de higiene no peridomicílio e a localização deste ao lado de pequenos capões de mata são freqüentemente observados nas áreas rurais em diversas regiões do Brasil (Gomes \& Neves, 1998; Lima, 2000; Teodoro, 1995; Teodoro et al., 2001a, $2001 b$ ). A soma destes fatores parece favorecer a concentração de flebotomíneos e de mamíferos reservatórios de Leishmania Ross no peridomicílio (Gomes \& Neves, 1998; Lima, 2000; Teodoro et al., 2001a, 2001b). Assim, as atividades de controle de flebotomíneos têm sido feitas utilizando-se a aplicação de inseticidas nos domicílios e em anexos existentes no peridomicílio (Gomes \& Neves, 1998; MS, 2000).

A importância vetorial dos flebotomíneos exige intensivas investigações da ecologia destes dípteros como parte integrante da rotina dos serviços de saúde pública, sobretudo nas regiões onde as leishmanioses são endêmicas. As pesquisas sobre flebotomíneos no Norte do Paraná vêm sendo realizadas em áreas endêmicas de leishmaniose tegumentar, em domicílios e peridomicílios de propriedades agrícolas onde já ocorreram casos desta doença (Lima, 2000; Teodoro, 1995; Teodoro \& Kühl, 1997; Teodoro et al., 2001a, 2001b).

Nesta investigação procurou-se avaliar o impacto da reorganização e limpeza do peridomicílio e da desinsetização de domicílios e abrigos de animais domésticos como medidas para diminuir a população de flebotomíneos no Recanto Marista, Município de Doutor Camargo, Estado do Paraná, visando o controle da leishmaniose tegumentar.

\section{Metodologia}

Este estudo foi desenvolvido no Recanto Marista, nas margens do rio Ivaí, numa área que comporta uma mata remanescente bastante alterada (Figura 1). Essa área fica no Município de Doutor Camargo que está inserido na Mesorregião Noroeste do Paraná, a 52¹3' Longitude Oeste e 23³3' Latitude Sul.

Os resultados de uma investigação realizada anteriormente no Recanto Marista (Teodoro et al., 2001a), de outubro de 1996 a setembro de 1997, são parcialmente resgatados nas Tabelas 1 e 2, para comparações com os dados obtidos nesta pesquisa. Após a investigação anterior (Teodoro et al., 2001a), diversas modificações (Figura 1) foram feitas no recanto, com a finalidade de reduzir a população de flebotomíneos, conforme se destaca em seguida:

- Limpeza de matéria orgânica acumulada no solo, representada especialmente por folhas e frutos em decomposição;

- Corte de algumas árvores ao lado da residência do administrador (E6) e da vegetação que formava um emaranhado entre as árvores localizadas ao redor da casa do administrador (E6) e do alojamento (E8);

- Drenagem do solo das áreas com elevada umidade, principalmente ao redor do curral, onde foi instalada a armadilha 3 (E3);

- Desinsetização de todas as edificações (residências, alojamentos, curral e galinheiros) com deltametrina (150mg/litro), em novembro de 1998 e março de 1999;

- Construção de galinheiros (E2, E5, E7, E9 e E10) nos quais uma lâmpada incandescente de 60W permanecia acesa, no período noturno, com a finalidade de atrair flebotomíneos, exceto nos dias de coletas.

As coletas de flebotomíneos foram realizadas de outubro de 1998 a abril de 2000, com armadilhas luminosas de Falcão, duas vezes ao mês, das 22 às 2 horas, totalizando 152 horas cada armadilha. O horário de coletas foi o mesmo utilizado por Teodoro et al. (2001a). As armadilhas foram instaladas em diversos ecótopos (E) conforme se vê na Figura 1 e se discrimina em seguida:

- Armadilha 1: na varanda da residência (E1) na entrada do Recanto Marista;

- Armadilha 2: dentro de uma pocilga acoplada a um galinheiro, nos quais sempre havia um suíno e vários frangos para abate; periodicamente o plantel de aves era renovado (E2);

- Armadilha 3: no interior de um curral, onde uma vaca e um bezerro permaneceram durante todo o período das coletas (E3); 
Figura 1

Distribuição das armadilhas de Falcão nos diversos ecótopos no Recanto Marista,

Município de Doutor Camargo, Paraná, Brasil.

Figura 1a

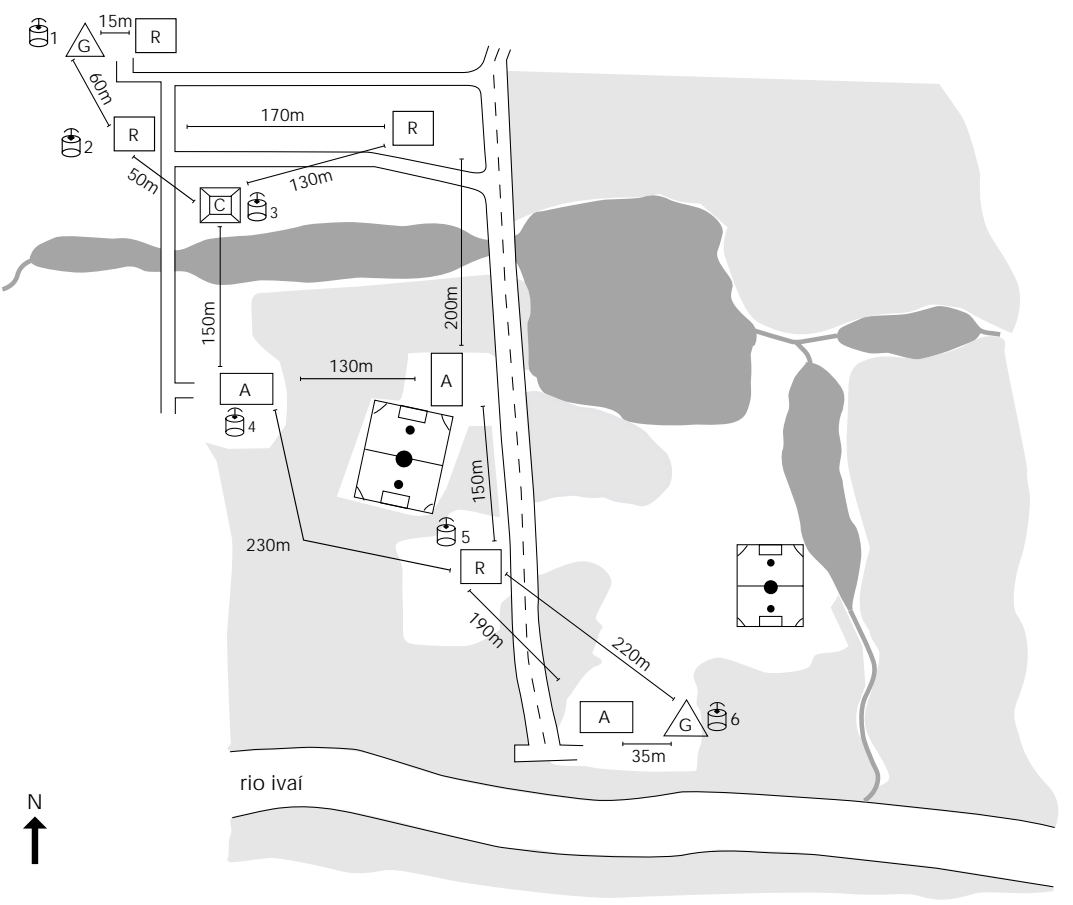

Figura 1b

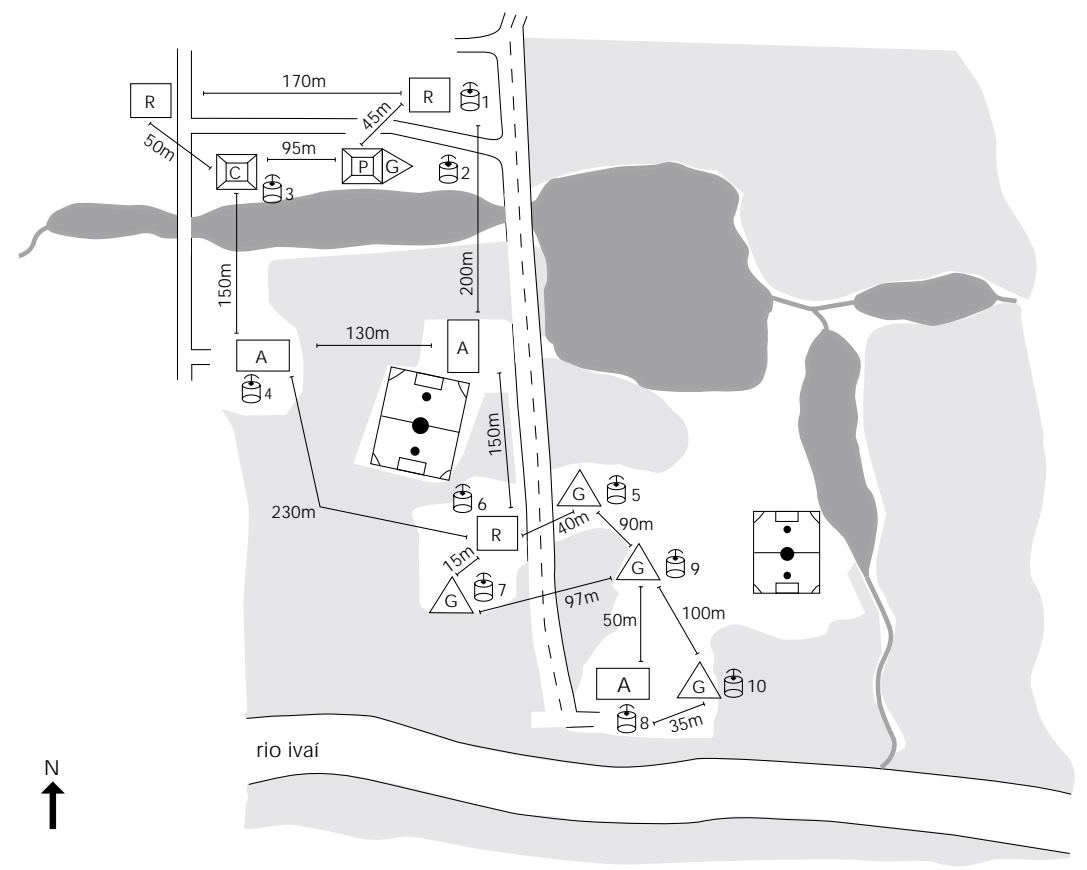

armadilha de falcão

G galinheiro

A alojamento

[C] curral

$\mathrm{R}$ residência

mata

lago
छิ armadilha de falcão

IP pocilga/galinheiro

(G) galinheiro

A alojamento

[C] curral

$\mathrm{R}$ residência

mata

lago 
Tabela 1

Fauna de flebotomíneos coletados no Recanto Marista, Município de Doutor Camargo, Paraná, de outubro de 1996 a setembro de 1997 e de outubro de 1998 a abril de 2000.

\begin{tabular}{|c|c|c|c|c|c|c|}
\hline \multirow[t]{2}{*}{ Espécie } & \multicolumn{3}{|c|}{ 1996/1997* } & \multicolumn{3}{|c|}{$1998 / 2000$} \\
\hline & Total & $\%$ & FA & Total & $\%$ & FA \\
\hline Lutzomyia neivai & 125.184 & 79,2 & 79,2 & 108.360 & 88,4 & 88,4 \\
\hline Lutzomyia whitmani & 28.678 & 18,2 & 97,4 & 13.666 & 11,1 & 99,5 \\
\hline Lutzomyia migonei & 2.799 & 1,8 & 99,2 & 354 & 0,3 & 99,8 \\
\hline Lutzomyia fischeri & 954 & 0,6 & 99,8 & 112 & 0,1 & 99,9 \\
\hline Lutzomyia pessoai & 125 & - & - & 34 & - & - \\
\hline Lutzomyia monticola & 27 & - & - & 10 & - & - \\
\hline Lutzomyia shannoni & 86 & - & - & 5 & - & - \\
\hline Lutzomyia cortelezzii & 15 & - & - & 8 & - & - \\
\hline Lutzomyia firmatoi & 1 & - & - & 1 & - & - \\
\hline Brumptomyia brumpti & 7 & - & - & 25 & - & - \\
\hline Brumptomyia cunhai & - & - & - & 5 & - & - \\
\hline Lutzomyia misionensis & - & - & - & 1 & - & - \\
\hline Lutzomyia sp. & 107 & - & - & 35 & - & - \\
\hline Total & 157.983 & & & 122.616 & & \\
\hline
\end{tabular}

$\mathrm{FA}=$ freqüência acumulada.

* Teodoro et al. (2001a).

Tabela 2

Média horária de flebotomíneos coletados no Recanto Marista, Município de Doutor Camargo, Paraná, de outubro de 1996 a setembro de 1997 e de outubro de 1998 a abril de 2000.

\begin{tabular}{|c|c|c|c|c|c|}
\hline \multicolumn{3}{|c|}{ 1996/1997* } & \multicolumn{3}{|c|}{$1998 / 2000$} \\
\hline Armadilha & Total & Média Horária & Armadilha & Total & Média Horária \\
\hline M 1 & 27.675 & 288,3 & $\mathrm{E} 1$ & 6.422 & 42,3 \\
\hline M2 & 3.332 & 34,7 & E2 & 5.155 & 33,9 \\
\hline M $3^{* *}$ & 78.710 & 819,8 & $\mathrm{E} 3^{* *}$ & 4.376 & 28,8 \\
\hline M 4** & 19.816 & 206,4 & $\mathrm{E} 4^{* *}$ & 9.268 & 61,0 \\
\hline - & & & E5 & 5.295 & 34,8 \\
\hline$M 5^{* *}$ & 22.733 & 236,8 & $\mathrm{E} 6^{* *}$ & 5.996 & 39,4 \\
\hline- & & & E7 & 10.054 & 66,1 \\
\hline M 6** & 5.359 & 55,8 & $E 8^{* *}$ & 4.066 & 26,8 \\
\hline- & & & E9 & 17.401 & 114,5 \\
\hline- & & & E10 & 54.583 & 359,1 \\
\hline Total & 157.625 & $1.641,9$ & Total & 122.616 & 806,7 \\
\hline
\end{tabular}

* Teodoro et al. (2001a).

** Estas armadilhas foram instaladas nos mesmos ecótopos em 1996/1997 e em 1998/2000.

Localização das armadilhas:

E1 - na varanda da residência na entrada de acesso ao Recanto Marista;

E2 - dentro de uma pocilga acoplada a um galinheiro; em todas as coletas sempre havia

um suíno e vários frangos para abate; periodicamente o plantel de aves era renovado;

E3 - no interior de um curral, com uma vaca e um bezerro, presentes durante to do o período de coletas;

E4 - em um anexo ao lado de uma residência onde, eventualmente, são alojados trabalhadores temporários;

E5 - num galinheiro onde eram mantidas de 7 a 10 galinhas, em todas as coletas;

E6 - numa varanda da residência do administrador do Recanto Marista;

E7 - num galinheiro onde eram criados de 20 a 25 cabeças de frangos para abate, com renovação periódica do plantel;

E8 - na entrada da porta principal de um alojamento, às margens do rio Ivaí,

usado por grupos de pessoas que procuram retiro espiritual;

E9 - num galinheiro onde eram mantidas de 7 a 10 galinhas, em todas as coletas;

E10 - num galinheiro onde eram mantidas de 7 a 10 galinhas, em todas as coletas. 
- Armadilha 4: em um anexo ao lado de uma residência onde, eventualmente, são alojados trabalhadores temporários (E4);

- Armadilha 5: num galinheiro com 7 a 10 galinhas, a uma distância de $40 \mathrm{~m}$ da residência do administrador (E5);

- Armadilha 6: na varanda da residência do administrador (E6);

- Armadilha 7: num galinheiro, distante $15 \mathrm{~m}$ da residência do administrador (E7), com 20 a 25 frangos para abate;

- Armadilha 8: na entrada principal de um alojamento, às margens do rio Ivaí, usado por grupos de pessoas que procuram retiro espiritual (E8);

- Armadilha 9: num galinheiro com 7 a 10 galinhas (E9);

- Armadilha 10: num galinheiro com 7 a 10 galinhas (E10).

Os ecótopos de coletas M3, M4, M5 e M6 da investigação anterior, de outubro de 1996 a setembro de 1997 (Teodoro et al., 2001a), correspondem aos ecótopos E3, E4, E6 e E8, respectivamente, desta investigação (Tabela 2). Nos outros (E1, E2, E5, E7, E9 e E10) as coletas foram feitas somente nesta investigação.

\section{Resultados}

As espécies coletadas, conforme a Tabela 1, foram: Brumptomyia brumpti (Larousse, 1920), B. cunhai (Mangabeira, 1942), Lutzomyia cortelezzii (Bréthes, 1924), L. firmatoi (Barreto, Martins \& Pellegrino, 1956), L. (Pintomyia) fischeri (Pinto, 1926), L. migonei (França, 1920), L. misionensis (Castro, 1959), L. monticola (Costa Lima, 1912), L. (Nyssomyia) neivai (Pinto, 1926), L. (Pintomyia) pessoai (Coutinho \& Barretto, 1940), L. (Psathyromyia) shannoni (Dyar, 1929), L. (Nyssomyia) whitmani (Antunes \& Coutinho, 1939).

Na Tabela 1 verifica-se que no período 1996/ 1997 (Teodoro et al., 2001a) foram coletados 157.983 flebotomíneos, representando dez espécies. L. neivai representou 79,2\% das espécies coletadas. Já no período 1998/2000 foram coletados 122.616 flebotomíneos de 12 espécies, das quais $L$. neivai representou $88,4 \%$. As espécies L. neivai, L. whitmani, L. migonei e $L$. fischeri representaram 99,9\% do total de flebotomíneos coletados.

Observa-se na Tabela 2 que a média de flebotomíneos coletados por hora (MH) em 1996/ 1997 foi 1.641,9 (Teodoro et al., 2001a), enquanto nesta investigação foi 806,7. As MH nos ecótopos E3, E4, E6 e E8 foram, respectivamente, 28,$8 ; 61,0 ; 39,4$ e 26,8. As MH anteriores (Teo- doro et al., 2001a) nos ecótopos M3, M4, M5 e M6 foram, respectivamente, 819,8; 206,4; 236,8 e 55,8. O maior número de flebotomíneos coletados foi $78.710(\mathrm{MH}=819,8)$ no ecótopo M3 no período 1996/1997 (Teodoro et al., 2001a), enquanto que no período 1998/2000, o maior número de flebotomíneos (54.583) foi coletado no ecótopo E10 (MH de 359,1).

L. neivai foi a espécie predominante em todo o período de coletas, tendo sido coletada em maior quantidade nos meses de janeiro, fevereiro, março e abril (Figura 2), representando $68,2 \%$ (73.874) dos exemplares. L. whitmani foi a segunda espécie predominante, com maior concentração em janeiro, fevereiro, agosto e dezembro de 1999 e abril de 2000, representando $58,6 \%$ (8.005) dos exemplares.

Comparando-se as Figuras 2 e 3, observa-se que, de um modo geral, as sazonalidades dos flebotomíneos nos diversos ecótopos de coletas foram semelhantes ao observado na somatória dos resultados das coletas desses ecótopos.

\section{Discussão}

Verificou-se que no conjunto das coletas nos diferentes ecótopos, a MH de flebotomíneos foi a metade neste estudo em relação a outro realizado anteriormente no mesmo local (Teodoro et al., 2001a). Considerando-se que no presente trabalho as coletas de flebotomíneos foram feitas em dez ecótopos (MH de 80,7 por ecótopo), contra seis no trabalho anterior, ( $\mathrm{MH}$ de 273,6 por ecótopo), coletaram-se 3,4 vezes menos flebotomíneos por ecótopo, após as modificações introduzidas no ambiente.

As precipitações pluviométricas observadas no período em que este trabalho foi realizado, de 1998 a 2000, acompanharam as médias normalmente observadas na região, segundo a Estação Climatológica do Departamento de Geografia da Universidade Estadual de Maringá. A sazonalidade dos flebotomíneos foi semelhante à que tem sido observada na região onde está inserida a área na qual foi feita esta investigação (Teodoro, 1995; Teodoro et al., 2001a), comprovando-se uma vez mais que nas épocas quentes e úmidas do ano coletam-se maior número de flebotomíneos. Anteriormente, as precipitações pluviométricas de outubro de 1996 a fevereiro de 1997 foram superiores às precipitações médias constatadas no período de 1988 a 1997, particularmente em janeiro e fevereiro de 1997 (Teodoro et al., 2001a). Em janeiro de 1997 houve alagamento de algumas áreas do Recanto Marista, onde foram feitas as coletas de flebotomíneos, e isto pode ter causado a re- 
Figura 2

Sazonalidade de Lutzomyia whitmani e L. neivai coletados no conjunto dos ecótopos no Recanto Marista,

Município de Doutor Camargo, Paraná, Brasil, de outubro de 1998 a abril de 2000.

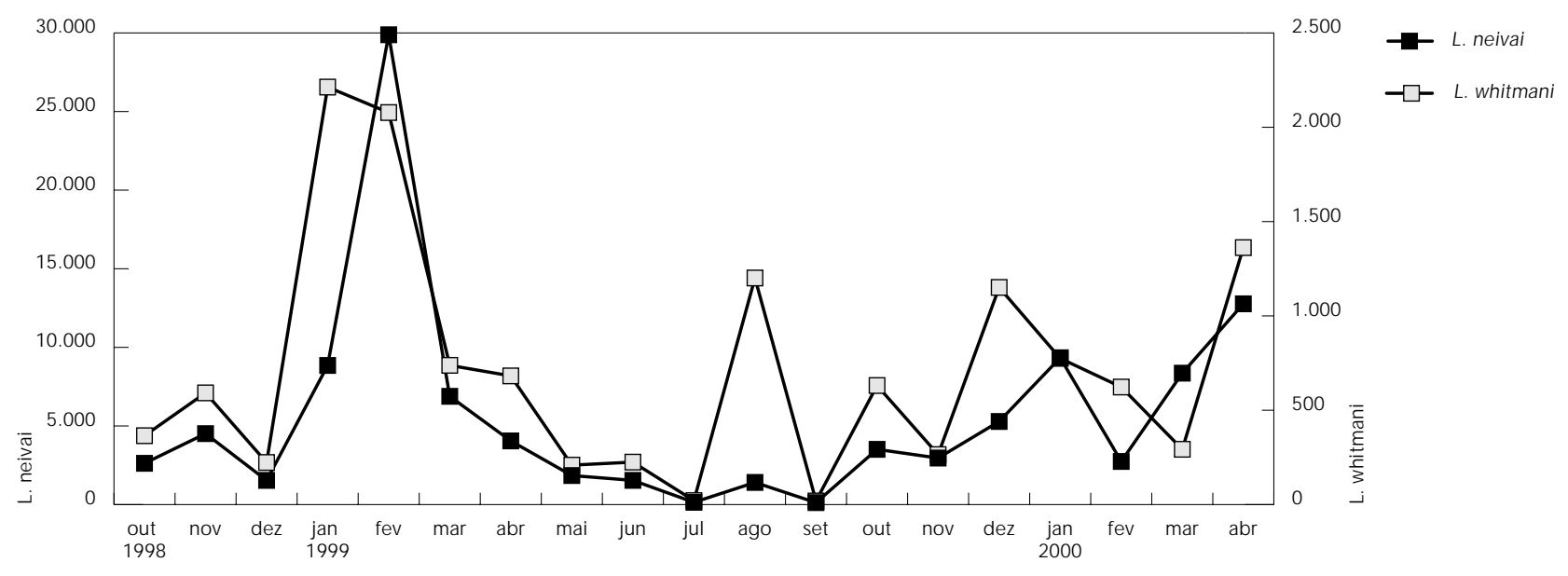

dução da população de flebotomíneos tanto neste mês quanto nos meses subseqüentes (Teodoro et al., 2001a).

Quando se comparam as MH de flebotomíneos coletados nas armadilhas instaladas nos ecótopos E3, E4, E6 e E8 com as MH dos ecótopos M3, M4, M5 e M6, do trabalho anterior (Teodoro et al., 2001a), observa-se que as $\mathrm{MH}$ neste estudo foram bem inferiores, especialmente no E3, que foi 28,5 vezes menor. Cabe lembrar que os ecótopos E3, E4, E6 e E8 correspondem aos ecótopos M3, M4, M5 e M6.

Nos ecótopos E3, E4, E6 e E8 foram coletados 19,3\% do total de flebotomíneos, enquanto que nos ecótopos M3, M4, M5 e M6, no período de 1996/1997 (Teodoro et al., 2001a), coletaram-se $80,7 \%$ dos flebotomíneos. A espécie L. neivai prevaleceu no decorrer de todo o período de coletas, assim como no trabalho anterior (Teodoro et al., 2001a). Contudo, a proporção desta espécie foi maior agora, provavelmente em função das alterações introduzidas no ambiente com o intuito de diminuir a população de flebotomíneos.

Embora L. neivai não tenha sido constatada com infecção por Leishmania spp., esta espécie tem predominado em áreas onde têm sido notificados casos de leishmaniose (Consolim et al., 1990; Teodoro \& Kühl, 1997), inclusive no Recanto Marista (Teodoro et al., 2001a), no Paraná. A prevalência de L. intermedia s.l. (L. neivai, segundo Marcondes, 1996, no Esta- do do Paraná), nos ambientes antrópicos, já foi observada por outros autores nos Estados de São Paulo (Gomes \& Neves, 1998) e Paraná (Teodoro \& Kühl, 1997; Teodoro et al., 2001a).

L. whitmani tem sido dominante e muito freqüente no peridomicílio de áreas endêmicas no Estado do Paraná (Luz et al., 2000; Teodoro et al., 1999, 2001a, 2001b), onde já se detectou esta espécie (Luz et al., 2000), com infecção por Leishmania (Viannia) braziliensis (Vianna, 1911). A importância de L. whitmani na epidemiologia da leishmaniose tegumentar foi relatada por Azevedo et al. (1990a) e Queiroz et al. (1994). Embora no Estado do Paraná não tenha sido constatada a infecção de L. migonei, sua importância foi demonstrada por Azevedo et al. (1990b), no Estado do Ceará e Camargo-Neves et al. (2002), no Estado de São Paulo.

Nos ecótopos construídos com a finalidade de atrair flebotomíneos (E2, E5, E7, E9 e E10) foram coletados $74,5 \%$ do total destes dípteros. O impacto provocado pelas desinsetizações, pela remoção de matéria orgânica (folhas e frutos) da superfície do solo e pela drenagem de algumas áreas pode ter contribuído para a redução da população de flebotomíneos (Teodoro et al., 1999). Tem sido demonstrado que nas áreas com solos úmidos e acúmulo de matéria orgânica de origem vegetal e animal há condições para a formação de criadouros e para a concentração de flebotomíneos no ambiente peridomiciliar (Casanova, 2001; Forattini, 1953; 
Figura 3

Sazonalidade do conjunto de espécies de flebotomíneos coletados em cada um dos ecótopos no Recanto Marista, Município de Doutor Camargo, Paraná, Brasil, de outubro de 1998 a abril de 2000.
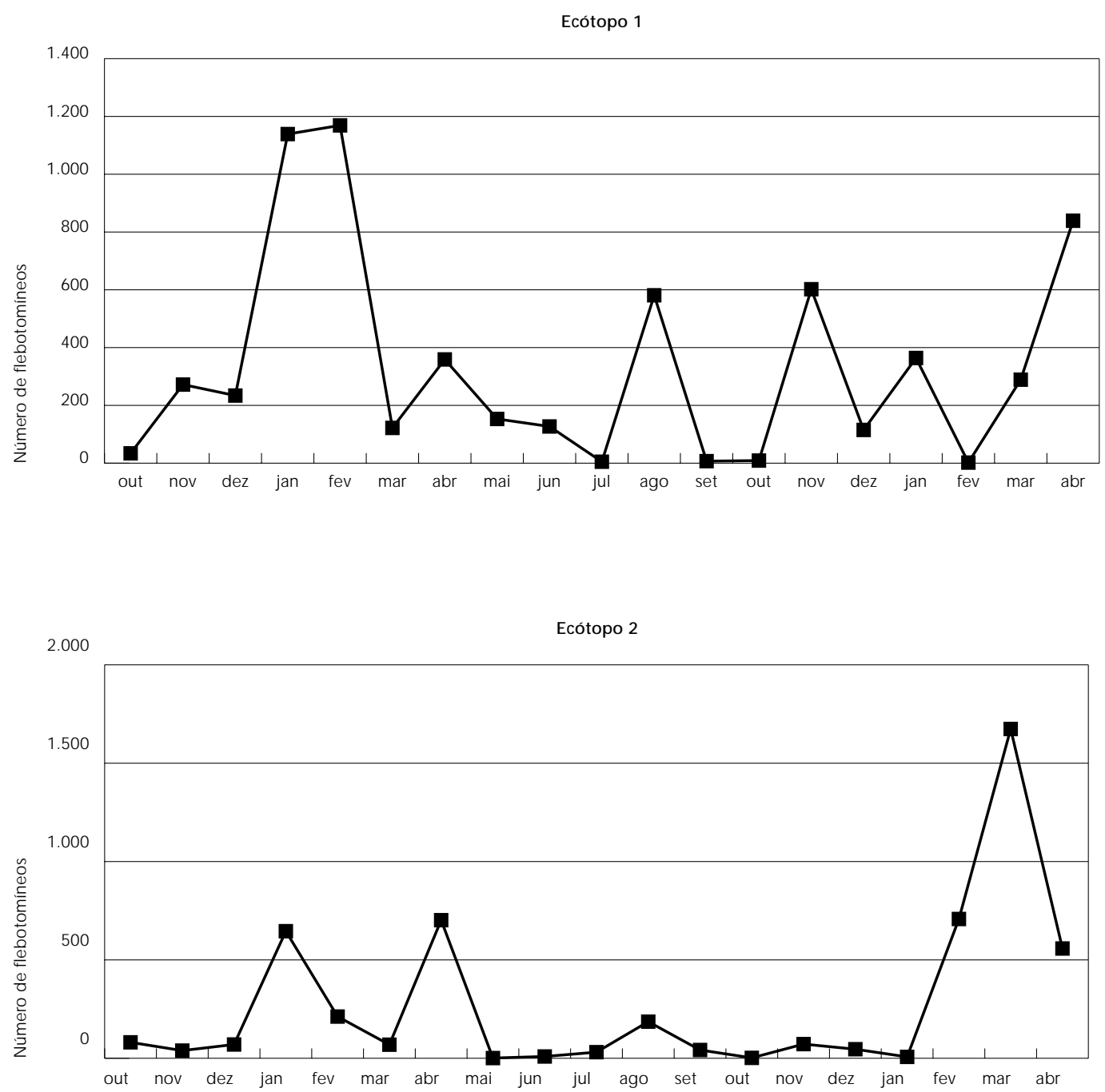

(continua) 
Figura 3 (continuação)

Sazonalidade do conjunto de espécies de flebotomíneos coletados em cada um dos ecótopos no Recanto Marista, Município de Doutor Camargo, Paraná, Brasil, de outubro de 1998 a abril de 2000.
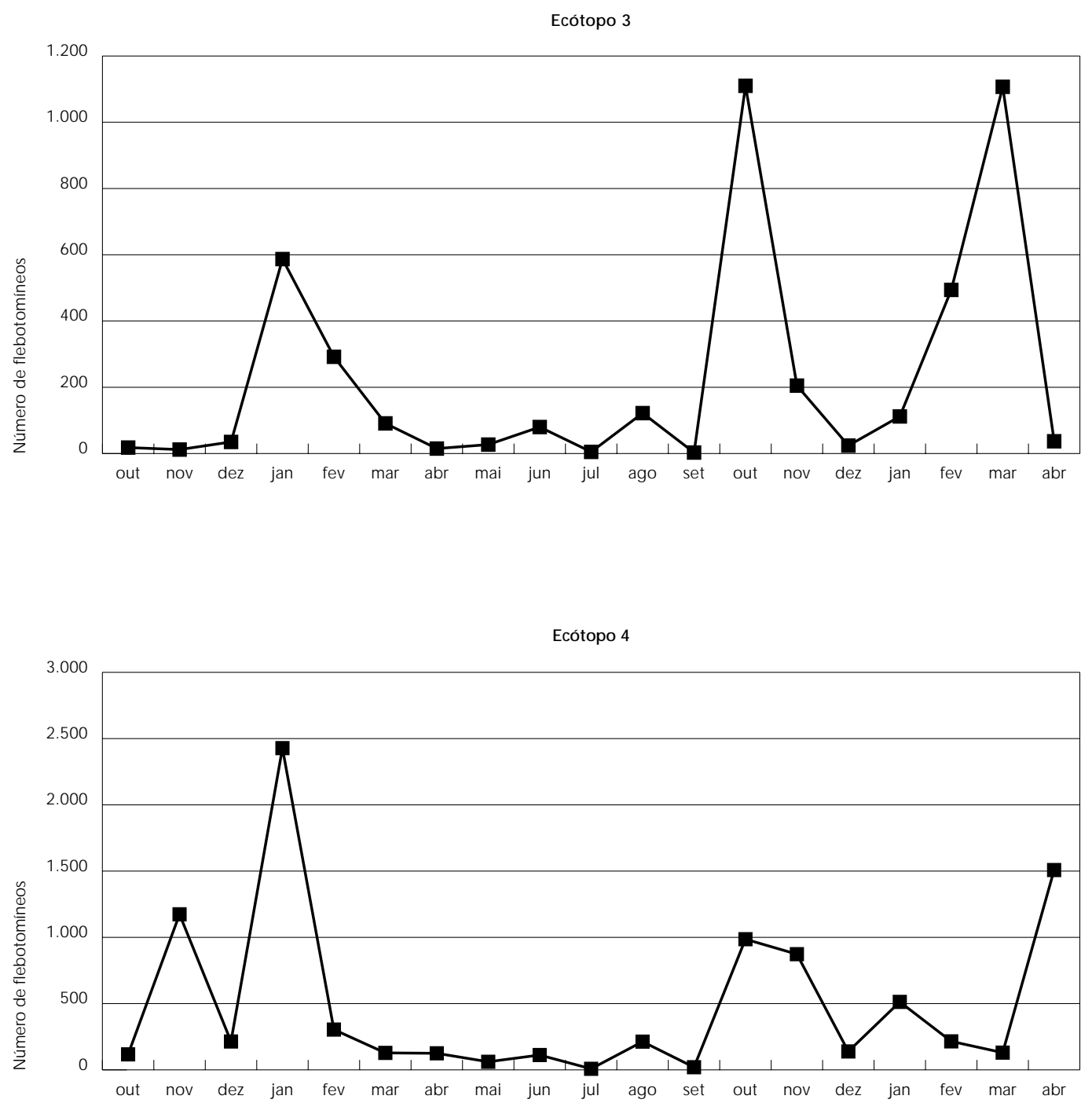

(continua) 
Figura 3 (continuação)

Sazonalidade do conjunto de espécies de flebotomíneos coletados em cada um dos ecótopos no Recanto Marista, Município de Doutor Camargo, Paraná, Brasil, de outubro de 1998 a abril de 2000.
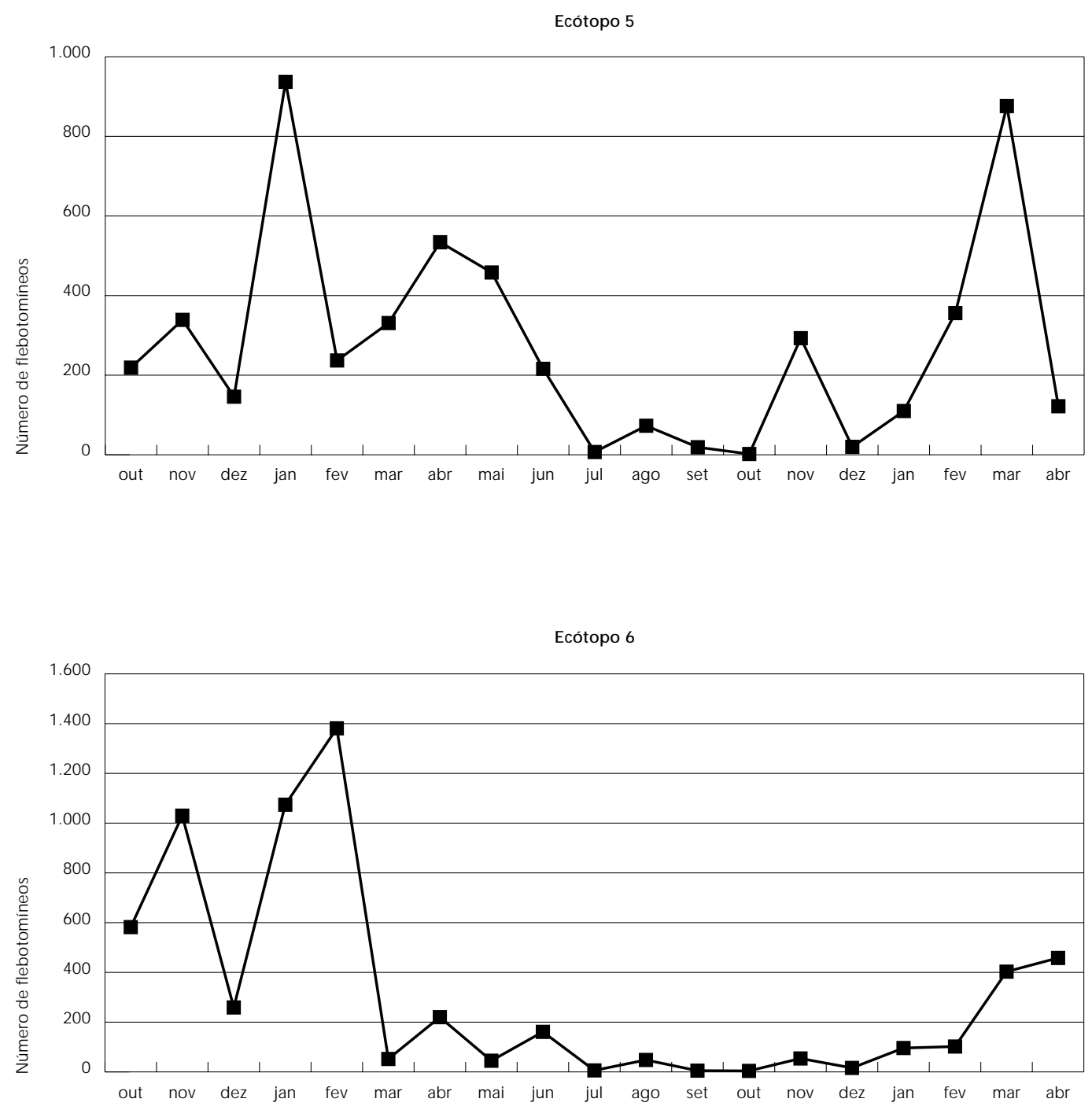

(continua) 
Figura 3 (continuação)

Sazonalidade do conjunto de espécies de flebotomíneos coletados em cada um dos ecótopos no Recanto Marista, Município de Doutor Camargo, Paraná, Brasil, de outubro de 1998 a abril de 2000.
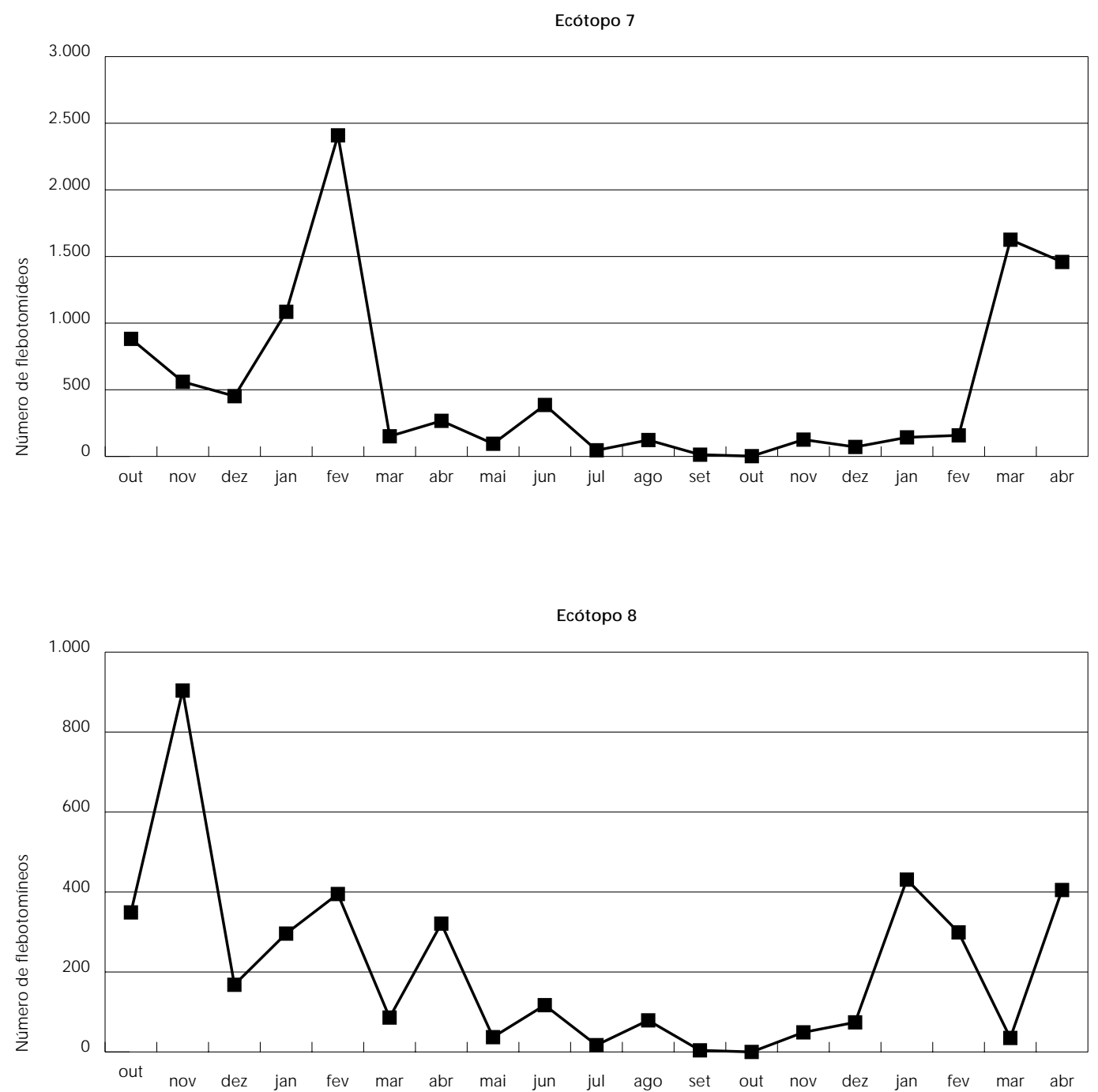

(continua) 
Figura 3 (continuação)

Sazonalidade do conjunto de espécies de flebotomíneos coletados em cada um dos ecótopos

no Recanto Marista, Município de Doutor Camargo, Paraná, Brasil, de outubro de 1998 a abril de 2000.
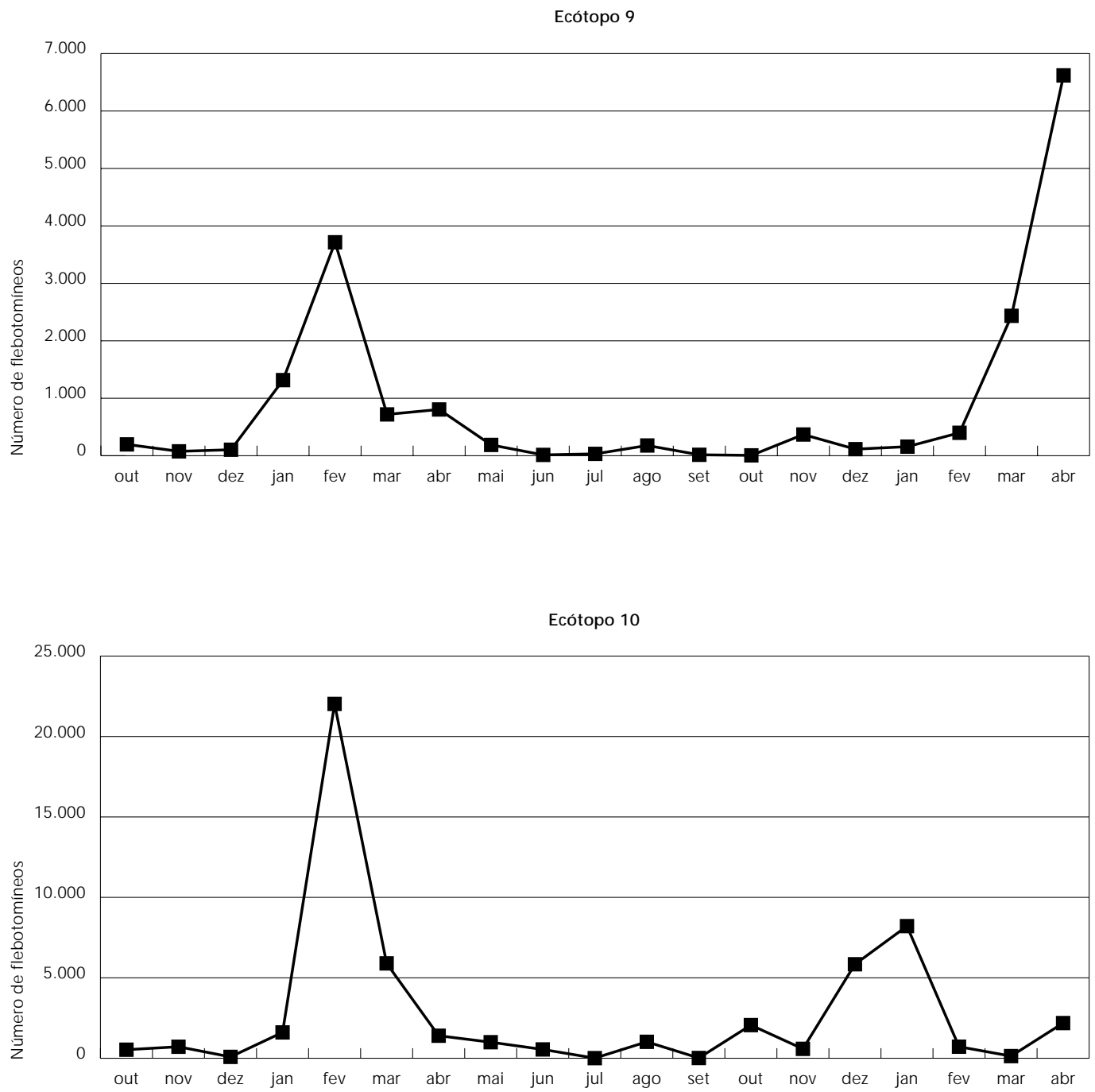
Souza et al., 1999; Teodoro et al., 1999; Vieira et al., 1999).

Observou-se neste trabalho que na primeira etapa nos ecótopos M3, M5 e M4 coletaramse o maior número de flebotomíneos $(76,3 \%)$ do total, enquanto na segunda, nos mesmos ecótopos (E3, E4 e E6) foram coletados 16,4\%. Contudo, na segunda etapa, nos ecótopos E7, E9 e E10, que foram construídos com a finalidade de atrair flebotomíneos, coletaram-se $66,9 \%$ do total. A desinsetização domiciliar e peridomiciliar tem demonstrado bons resultados no controle da população de flebotomíneos (Alencar, 1961; Deane et al., 1955; Sherlock \& Almeida, 1970; Teodoro et al., 1998). As medidas de controle de flebotomíneos adotadas nesta investigação foram aplicadas anteriormente por Teodoro et. al. (1999), contribuindo para reduzir a densidade de flebotomíneos no domicílio e no peridomicílio. Assim, acredita-se que tanto a desinsetização quanto a reorganização do ambiente contribuíram para o controle da densidade de flebotomíneos.

\section{Considerações finais}

Houve uma redução expressiva no número de flebotomíneos coletados de outubro de 1998 a abril de 2000, comparado ao de outubro de 1996 a setembro de 1997, possivelmente como conseqüência do impacto causado pelas alte- rações no ambiente e pela desinsetização das edificações, logo após este período de coletas. A redução da população de flebotomíneos no ambiente peridomiciliar e no domicílio pode ter contribuído para diminuir o risco de transmissão de Leishmania para o homem e para os animais domésticos, no Recanto Marista. Cabe lembrar que neste recanto, antes das medidas adotadas, foram notificados dez casos de leishmaniose (cinco mulheres adultas, quatro homens adultos e uma criança do sexo masculino) entre 24 moradores, além de nove cães com sorologia positiva por imunofluorescência indireta, sendo dois com exame parasitológico positivo (Teodoro et al., 2001a). Durante a realização deste trabalho, nenhum caso de leishmaniose foi notificado. Contudo, no ano de 2001 ocorreu um caso de leishmaniose nesta localidade, conforme diagnóstico realizado no Laboratório de Ensino e Pesquisa em Análises Clínicas da Universidade Estadual de Maringá, possivelmente em função do relaxamento na manutenção das medidas aplicadas para diminuir a população de flebotomíneos no domicílio e nas suas imediações.

A mudança nas proporções das espécies de flebotomíneos, especialmente de $L$. neivai, foi possivelmente em decorrência das alterações introduzidas no ambiente, tendo em vista a melhor adaptação desta espécie nos ambientes com maior grau de antropia, em detrimento de L. whitmani e das demais espécies.

\section{Referências}

ALENCAR, J. E., 1961. Profilaxia do calazar no Brasil no Ceará, Brasil. Revista do Instituto de Medicina Tropical de São Paulo, 3:175-180.

AZEVEDO, A. C. R.; RANGEL, E. F.; COSTA, E. M.; DAVID, J.; VASCONCELOS, A. W. \& LOPES, U. G., 1990a. Natural infection of L. (Nyssomyia) whitmani (Antunes \& Coutinho, 1939) by Leishmania of the braziliensis complex in Baturité, Ceará State, Northeastern Brazil. Memórias do Instituto Oswaldo Cruz, 85:251.

AZEVEDO, A. C. R.; RANGEL, E. F. \& QUEIROZ, R. G., 1990b. L. migonei (França, 1920) naturally infected with peripylarian flagellates in Baturité, a focus of cutaneous leishmaniasis in Ceará State, Brazil. Memórias do Instituto Oswaldo Cruz, 85: 479.
CAMARGO-NEVES, V. L. F.; GOMES, A. C. \& ANTUNES, J. L. F., 2002. Correlação da presença de espécies de flebotomíneos (Diptera: Psychodidae) com registros de casos da leishmaniose tegumentar americana no Estado de São Paulo, Brasil. Revista da Sociedade Brasileira de Medicina Tropical, 35:299-306.

CASANOVA, C., 2001. A soil emergence trap for collections of phlebotomine sand flies. Memórias do Instituto Oswaldo Cruz, 92:273-275.

CONSOLIM, J.; LUZ, E. \& TORRES, P. B., 1990. Flebótomos da área do reservatório da hidroelétrica de Itaipu, Estado do Paraná, Brasil (Diptera, Psychodiade, Phlebotominae). Cadernos de Saúde Pública, 6:74-85. 
DEANE, L. M.; DEANE, M. P. \& ALENCAR, J. E., 1955. Observações sobre o combate ao Phlebotomus longipalpis pela dedetização domiciliária em focos endêmicos de calazar, Ceará. Revista Brasileira de Malariologia e Doenças Tropicais, 7:131-141.

FORATTINI, O. P., 1953. Nota sobre criadouros naturais de flebótomos em dependências peridomiciliares, no Estado de São Paulo. Arquivos da Faculdade de Higiene de São Paulo, 7:157-165.

GOMES, A. C. \& NEVES, V. L. F. C., 1998. Estratégia e perspectiva de controle da leishmaniose tegumentar no Estado de São Paulo. Revista da Sociedade Brasileira de Medicina Tropical, 6:553558.

LIMA, A. P., 2000. Distribuição da Leishmaniose Tegumentar e Análise da sua Ocorrência em Ambientes Antrópicos, no Estado do Paraná, Brasil. Dissertação de Mestrado, Londrina: Universidade Estadual de Londrina.

LUZ, E.; MEMBRIVE, N.; CASTRO, E. A.; DEREURE, J.; PRATLONG, J.; DEDET, A.; PANDEY, A. \& THOMAZSOCCOL, V., 2000. Lutzomyia whitmani (Díptera: Psychodidae) as vector of Leishmania (V.) braziliensis in Paraná State, Southern Brazil. Annals of Tropical Medicine and Parasitology, 94:623631.

MARCONDES, C. B., 1996. A redescription of Lutzomyia (Nyssomyia) intermedia (Lutz \& Neiva, 1912), and ressurection of L. neivai (Pinto, 1926) (Diptera, Psychodidae, Phlebotominae). Memórias do Instituto Oswaldo Cruz, 91:457-462.

MS (Ministério da Saúde), 1997. Série histórica de casos de agravos e doenças infecciosas e parasitáriasBrasil-1980 a 1996. Informe Epidemiológico do SUS, 6:59-60.

MS (Ministério da Saúde), 2000. Manual de Controle da Leishmaniose Tegumentar Americana. Brasília: MS.

QUEIROZ, R.; VASCONCELOS, I. A. B.; VASCONCELOS, A. W.; PESSOA, F. A. C.; SOUSA, R. N. \& DAVID, J. R., 1994. Cutaneous leishmaniasis in Ceará in Northeastern Brazil: incrimination of Lutzomyia whitmani (Diptera: Psychodidae) as a vector of Leishmania braziliensis in Baturité municipality. American Journal of Tropical Medicine and Hygiene, 50:693-698.

SHERLOCK, I. A. \& ALMEIDA, S. P., 1970. Observações sobre o calazar em Jacobina, Bahia. V. Resultados de medidas profiláticas. Revista Brasileira de Malariologia e Doenças Tropicais, 22:175-182.
SOUZA, R. N.; LIMA, J. W. O.; SOUZA, F. V.; GADELHA, A. C. \& BRAGA, V. S. S., 1999. Estabelecimento de um criadouro natural de Lutzomyia longipalpis. In: XXXV Congresso da Sociedade Brasileira de Medicina Tropical, Resumos, pp. 214-215, Guarapari: Sociedade Brasileira de Medicina Tropical.

TEODORO, U., 1995. Características Ecológicas de Flebotomineos (Diptera, Psychodidae) em Habitats Antrópicos, Município de Jussara, Paraná, Brasil. Tese de Doutorado, Curitiba: Universidade Federal do Paraná.

TEODORO, U.; GALATI, E. A. B.; KÜHL, J. B.; LOZOVIE, A. L. \& BARBOSA, O. C., 1998. Controle de flebotomíneos com DDT, em área endêmica de leishmaniose tegumentar no Estado do Paraná, sul do Brasil. Brazilian Archives of Biology and Technology, 41:359-364.

TEODORO, U. \& KÜHL, J. B., 1997. Interação flebotomíneos, animais domésticos e dominância de Lutzomyia (Nyssomyia) intermedia (Lutz \& Neiva, 1912) em área com alto grau de antropia, no sul do Brasil. Revista de Saúde Pública, 31:512516.

TEODORO, U.; KÜHL, J. B.; ABBAS, M. \& DIAS, A. C., 2001 b. Luz e aves como atrativos de flebotomíneos (Diptera, Psychodidae), no sul do Brasil. Revista Brasileira de Entomologia, 45:167-172.

TEODORO, U.; KÜHL, J. B.; THOMAZ-SOCCOL, V.; BARBOSA, O. C.; FERREIRA, M. E. M. C.; LOZOVEI, A. L.; VERZIGNASSI, T. G. \& ROBERTO, A. C. B. S., 1999. Environmental sanitation and peridomiciliar organization as auxiliary practices for the control of phlebotomines in Paraná State, Southern Brazil. Brazilian Archives of Biology and Tecnology, 42:307-314.

TEODORO, U.; SILVEIRA, T. G. V.; SANTOS, D. R.; SANTOS, E. S.; SANTOS, A. R.; OLIVEIRA, O. \& KÜHL, J. B., 2001a. Freqüência da fauna de flebotomíneos no domicílio e em abrigos de animais domésticos no peridomicílio, nos municípios de Cianorte e Doutor Camargo - Estado do Paraná - Brasil. Revista de Patologia Tropical, 30 : 209-233.

VIEIRA, V. P.; FERREIRA, A. L. \& FALQUETO, A., 1999. Pesquisa de criadouros de flebotomíneos no ambiente peridomiciliar, em área endêmica de leishmaniose tegumentar (LT) no Estado do Espírito Santo. In: XXXV Congresso da Sociedade Brasileira de Medicina Tropical, Resumos, p. 31, Guarapari: Sociedade Brasileira de Medicina Tropical.

Recebido em 26 de julho de 2002

Versão final reapresentada em 21 de maio de 2003

Aprovado em 12 de agosto de 2003 